\title{
Physical, Physicochemical, Mechanical, and Structural Characterization of Films Based on Gelatin/Glycerol and Carbon Nanotubes
}

\author{
Israel Sifuentes-Nieves, ${ }^{1}$ Rodolfo Rendón-Villalobos, ${ }^{1}$ \\ Antonio Jiménez-Aparicio, ${ }^{1}$ Brenda Hildeliza Camacho-Díaz, ${ }^{1}$ \\ Gustavo Fidel Gutiérrez López, ${ }^{2}$ and Javier Solorza-Feria ${ }^{1}$ \\ ${ }^{1}$ Instituto Politécnico Nacional, CEPROBI, Calle Ceprobi No. 8, Colonia San Isidro, 62731 Yautepec, MOR, Mexico \\ ${ }^{2}$ Instituto Politécnico Nacional, ENCB, Carpio y Plan de Ayala, Delegación M. Hidalgo, 11340 México, DF, Mexico \\ Correspondence should be addressed to Javier Solorza-Feria; jsolorza@ipn.mx
}

Received 4 July 2014; Revised 15 April 2015; Accepted 16 April 2015

Academic Editor: Peng He

Copyright ( 2015 Israel Sifuentes-Nieves et al. This is an open access article distributed under the Creative Commons Attribution License, which permits unrestricted use, distribution, and reproduction in any medium, provided the original work is properly cited.

\begin{abstract}
A new method to prepare glycerol/gelatin based films, by doping the film with carbon nanotubes (CNTs) and sodium dodecyl sulfate (SDS), was proposed. SDS was used to disperse CNTs in gelatin/glycerol films as follows: gelatin/glycerol (GG) incubated with equal concentrations of CNT and SDS; GG with $0.001 \%$ w/w CNT/SDS; GG with $0.002 \%$ CNT/SDS and GG with $0.004 \%$ CNT/SDS. Diffractograms of CNT/SDS /glycerol films showed an amorphous structure, being consistent with thermograms involving temperature and fusion enthalpy. Mechanical tests showed 30\% increase in elongation at break of GG with $0.004 \%$ CNT/SDS, with respect to gelatin/glycerol/SDS control. Samples with CNT had increased water vapor permeability (WVP). The film fractal dimension indicated that, with the addition of the highest concentration of CNT, films with a homogeneous surface were obtained, with probable nanotube inclusion in the protein matrix. According to the results, the easy method used to prepare gelatin composite materials gave place to films with better physical, mechanical, and thermal properties.
\end{abstract}

\section{Introduction}

The resistance to corrosion, weather, and degradation by microorganisms and key physical properties of synthetic plastics currently has a major impact on environmental pollution [1]. As a result, there has been increasing interest in developing biodegradable packaging. Gelatin is an attractive biopolymer with several potential benefits, including low production cost on a large scale and easy degradation [2]. However, biopolymers generally have several mechanical properties that must be improved to enable their use in various applications [3]. Arvanitoyannis et al. [4] found that films made from gelatin are fragile and susceptible to cracking due to the high density of the cohesive energy of the polymer. Studies to date have described the effects of plasticizers in the physical, chemical, and functional properties of biofilms, since reduced intermolecular bonds allow greater flexibility in their structures. Most of these plasticizers are low molecular weight compounds that can easily integrate into the structure of macromolecules forming the film [5]. Similarly, it has been observed that films produced from proteins and plasticizers present disadvantages with respect to their mechanical and barrier properties, which have resulted in the use of mixtures of two or more compounds to improve the functional properties of their biodegradable films [6]. It has also been reported that the use of nanoparticles can improve the mechanical, thermal, and electrical properties of biodegradable polymers [7]. Among these, single wall carbon nanotubes (CNTs) are described as a twodimensional rolled layer of graphite that forms a cylinder that is tenths of microns in length with a radius in the order of nanometers. Their extremes possess fullerene semistructures. Multiple walls (MWCNT) are formed by concentric layers of cylindrical shapes, which are spaced approximately at 
a distance similar to the interplanar distance of graphite [8]. The elastic deformation exhibited by the CNTs makes them extraordinarily resistant materials, and their ability to maintain loads at large angles of bending allows them to store or absorb significant amounts of energy. CNTs have exceptional mechanical, thermal, and physical properties, which make them good candidates for the development of reinforced composite materials. Furthermore, CNTs have the ability to withstand large deformations without appreciable damage to the structure, even when they are combined with other components [9]. However, the main obstacles in biological applications of CNTs are their biocompatibility and low solubility in aqueous solutions, which is why solvents must be employed to maximize proper dispersion for reinforcement [7]. Since the inclusion of carbon nanotubes into polymer matrices has been shown to remarkably improve the mechanical properties of the resulting composites and to provide overall better functional properties and as far as we are concerned not enough studies on reinforcement of gelatin composites have been reported, the aim of this study was to evaluate the effect of the incorporation of carbon nanotubes in the physical, physicochemical, structural, and mechanical properties of films prepared from a gelatin/glycerol/SDS composite, using a method readily available.

\section{Experimental}

2.1. Materials. For the preparation of the films, $225^{\circ}$ Bloom (Sigma-Aldrich, USA) commercial gelatin of animal origin (type B) was used. Glycerol (Fermont, Mexico) was used as a plasticizer. CNTs with multiwalled characteristics (MWCNT) synthesized by the chemical vapor deposition (CVD) technique were purchased from Arkema (Lacq, France). Diameter is equal to $22.40 \pm 2.62 \mathrm{~nm}$ and is dispersed with sodium dodecyl sulfate (SDS) (Reasol, Mexico). All commercially available chemical reagents were of reagent grade and were used as received without further purification.

2.2. CNT Dispersion with SDS in Aqueous Medium. For the dispersion of CNTs, the method proposed by Sánchez [7] was used with some modifications. Solutions were prepared with equal concentrations of both CNT and SDS $(0.001,0.002$, and $0.004 \% \mathrm{w} / \mathrm{w}$ ) in $20 \mathrm{~mL}$ of deionized water, which were kept in an ultrasonic bath (BRANSON, Mexico) for $4 \mathrm{~h}$ at $50.0^{\circ} \mathrm{C}$.

2.3. Preparation of Gelatin Films. Films with four different formulations were made: control GG or gelatin/glycerol/SDS control with no nanotubes addition; GGCNT1 or gelatin/ glycerol/SDS and CNT at $0.001 \%(0.016 \mathrm{~g}$ CNT/100 g gelatin); GGCNT2 or gelatin/glycerol/SDS and CNT at $0.002 \%(0.032 \mathrm{~g} \mathrm{CNT} / 100 \mathrm{~g}$ gelatin); and GGCNT4 or gelatin/glycerol/SDS and CNT at $0.004 \% \mathrm{w} / \mathrm{w}(0.066 \mathrm{~g}$ $\mathrm{CNT} / 100 \mathrm{~g}$ gelatin), respectively. A solution of gelatin $(3 \% \mathrm{w} / \mathrm{w}$, previously hydrated) and glycerol $(1 \% \mathrm{w} / \mathrm{w})$ was prepared, to which the CNTs dispersed at the concentrations previously mentioned were added. The prepared solutions were then sonicated for $2 \mathrm{~h}$ according to the methodology proposed by Haider et al. [10] and were emptied into sterile polystyrene Petri dishes $(114 \times 20 \mathrm{~mm})$. The suspensions of the biopolymer-glycerol-nanocomposite were dried at $60.0^{\circ} \mathrm{C}$ in an oven for $48 \mathrm{~h}$, and the films produced were stored in a desiccator at room temperature, with a constant relative humidity (RH) of $57.0 \%$, conferred by a saturated solution of sodium bromide ( $\mathrm{NaBr}$ ) (Fermont, Mexico).

2.4. Conditioning Film. Prior to analysis, the films were conditioned for 7 days in a desiccator containing $\mathrm{NaBr}$ at a regulated temperature of $25.0 \pm 2.0^{\circ} \mathrm{C}$ and $\mathrm{RH}$ of $57.0 \pm 2.0 \%$ [11].

2.5. Color Determination. The color of the films was determined using a Universal Colorimeter (Milton-Roy, Color Matte, USA), with a D65 illuminant and a fixed observation angle of $10.5^{\circ}$. Five readings were taken for each sample. The equipment was calibrated previously with a standard of white color. The readings were reported in the CIELAB system $\left(L^{*}, a^{*}, b^{*}\right)$ for this study with consideration of the brightness value $\left(L^{*}\right)$.

2.6. Moisture Content Determination. The moisture content of the films was determined according to the AOAC 14.004 method [12], by measuring the loss in weight of the films after drying them in an oven at $110.0^{\circ} \mathrm{C}$ for $2 \mathrm{~h}$. Samples were analyzed in triplicate and the results are expressed as a percentage of moisture.

2.7. Water Activity $\left(A_{W}\right)$ Determination. To assess the water activity of the film, a water activity meter (AQUA LAB 4TE, USA) was used. Measurements were performed in triplicate for each sample according to the AOAC 978.18 method [12].

2.8. Solubility Percentage Determination. The solubility percentage of the films was determined according to the methodology proposed by García et al. [11]. Parts of each film were cut and stored in a desiccator at RH close to $0.0 \%$ for 7 days. The samples were then weighed and immersed in deionized water. The samples were constantly agitated $(125 \mathrm{rpm})$ for $1 \mathrm{~h}$ at room temperature and at boiling temperature $\left(80.0^{\circ} \mathrm{C}\right)$. Pieces of the films were then dried in an oven at $60.0^{\circ} \mathrm{C}$ for $2 \mathrm{~h}$. The results represent the average of three determinations.

2.9. X-Ray Diffraction. The diffractograms were obtained on a Siemens D5000 diffractometer operated with a voltage of $40 \mathrm{kV}$ and a current of $30 \mathrm{~mA}$, which was equipped with a $\mathrm{Cu} \mathrm{K}$ alpha radiation line. A scan was made in an angular range of $3-40^{\circ}(2 \theta)$ with an angular step of $0.05^{\circ}$ in a counting time per angular step of $15 \mathrm{~s}$. The crystallinity percentage was calculated by the method of Hayakawa et al. [13] and Manful et al. [14] wherein the total area of the peak of crystallinity of the diffraction pattern was used with the baseline calculated to be between 8 and $30^{\circ}$.

2.10. Thermal Properties. For the determination of the thermal properties of the films, a TA 2010 differential scan calorimeter (DSC) (TA Instruments, New Castle, DE) was used. The fusion temperature $\left(T_{f}\right)$ and enthalpy $(\Delta H)$ of the films were determined by heating the sample from room temperature to $250.0^{\circ} \mathrm{C}$. The glass transition temperature $\left(T_{g}\right)$ was also determined in the films by taking $2.1 \pm 0.03 \mathrm{mg}$ of the samples and placing them in aluminum trays, which 
were sealed by a mechanical press. The measurement was performed twice by first cooling the film sample to $-150.0^{\circ} \mathrm{C}$ and then heating it to $250.0^{\circ} \mathrm{C}$. Thermal parameters were calculated from the results of the second run using TA Instruments Universal Analysis 2000 software. This entire procedure was performed three times for each sample.

2.11. Mechanical Properties. The mechanical tests consisted of trials to determine tensile strength or tension fracture $(\mathrm{TF})$, elongation at break or elongation percentage $(\% E)$, and modulus of elasticity (ME). To determine the resistance of the film to tension, testing was performed according to the D-882-95a standard method of ASTM [15] on TAXT2i texture equipment (Stable Micro Systems, Surrey, UK) using a cell load of $25 \mathrm{Kg}$. For this determination, the film samples were cut into rectangles that were $10.0 \mathrm{~cm}$ long and $1.0 \mathrm{~cm}$ wide. The spacing between the clamps was $6.0 \mathrm{~cm}$ (effective distance of test). Then, $2.0 \mathrm{~cm}$ of the cut film was placed in each of the clamps. A strain rate of $24.0 \mathrm{~mm} \mathrm{~min}^{-1}$ was used. The tension fracture was calculated as the ratio of the maximum force on the film during the break between the cross sectional areas (thickness by width). The elongation percentage at the fracture was calculated by measuring the maximum length of the film between the final and initial separation of the clamps. The elastic modulus was calculated from the slope that was formed during the linear behavior of the stress-strain curve.

2.12. Water Vapor Permeability (WVP). The water vapor permeability (WVP) of the films was determined by the modified E-96-80 method [16]. The films were conditioned in a cell with $\mathrm{RH}$ of $57.0 \%$ conferred by saturated $\mathrm{NaBr}$ and placed so that the top of the permeation cell was sealed on a circular opening of $0.0005439 \mathrm{~m}^{2}$. To maintain $\mathrm{RH}$ gradient through the films, silica gel was placed into the permeation cell $(0.11 \%)$ and the cells were placed in a desiccator, which itself contained a saturated solution of $\mathrm{NaCl}(75.0 \% \mathrm{RH})$. The increase in weight was determined gravimetrically every hour until equilibrium was reached. Once this balance was achieved, the film thickness was measured to calculate the WVP. The results correspond to the average of three determinations.

2.13. Image Analysis. Images were captured using a stereoscopic microscope (NIKON SMZ 1500 model, Japan) coupled to a digital camera (3CCD MTI model, Dage-MTI Inc., Michigan, USA) using illumination with transmitted light, $1.5 \mathrm{x}$ objective lens with $640 \times 480$ image resolution, and TIF image format. Thirty micrographs were taken for each sample to measure texture parameters, as the percentage of area $(\% A)$ occupied by carbon nanotubes on the film area, as well as the entropy using GLCM texture plugins and surface fractal dimension (SFD) with the box counting method. The images were captured using Metamorph V.6.1r0 and then, these were processed in ImageJ software (NIH Image, Bethesda, Maryland, USA).

2.14. Statistical Analysis. An analysis of variance (ANOVA) at a level of significance of $5 \%(P<0.05)$ was applied. The
TABLE 1: Luminosity factor $\left(L^{*}\right)$, moisture content, and water activity of films from gelatin/glycerol and carbon nanotubes* .

\begin{tabular}{lccc}
\hline Sample & Luminosity & \% moisture & $A_{W}$ \\
\hline GG & $84.50 \pm 0.40^{\mathrm{a}}$ & $14.38 \pm 0.17^{\mathrm{a}}$ & $0.36 \pm 0.01^{\mathrm{a}}$ \\
GGNTC1 & $61.10 \pm 0.28^{\mathrm{b}}$ & $14.35 \pm 0.60^{\mathrm{a}}$ & $0.37 \pm 0.01^{\mathrm{a}}$ \\
GGNTC2 & $42.29 \pm 0.15^{\mathrm{c}}$ & $14.21 \pm 0.09^{\mathrm{a}}$ & $0.37 \pm 0.01^{\mathrm{a}}$ \\
GGNTC4 & $39.02 \pm 0.63^{\mathrm{d}}$ & $14.53 \pm 0.40^{\mathrm{a}}$ & $0.37 \pm 0.01^{\mathrm{a}}$ \\
\hline
\end{tabular}

${ }^{*}$ Mean values of three measurements \pm standard error. GG: gelatin/glycerol/SDS control film; GGNTC1, GGNTC2, and GGNTC4: films with gelatin/glycerol/SDS/CNT. Same letters after entries within each column indicate that there are no significant differences $(P>0.05)$.

Sigma Plot statistical package, version 11.0, was used for all calculations. When significant differences between treatment means were found, Tukey's multiple comparison test [17] was applied.

\section{Results and Discussion}

3.1. Color. The evaluation of color is important since it can be seen as a quality parameter for potential industrial applications of the films. Significant differences $(P<0.05)$ between the luminosity value $\left(L^{*}\right)$ of all films produced (Table 1) were observed. The GG or gelatin/glycerol/SDS control film showed a value of $L^{*}$ that tended to be white (84.50). This result was similar to that reported by Vazquez [18], who obtained $L^{*}$ value of 84.20 in films prepared from fish gelatin. Remarkably, the transparency of the films made from gelatin depended on the source from which it was obtained (e.g., beef, pork, fish, or poultry) and gel strength (Bloom). In general, the control film presented the highest $L^{*}$ value, while the addition of different amounts of CNTs made the $L^{*}$ value decrease, with the GGCNT4 film having the lowest value (Table 1).

The CNT concentration reduced the $L^{*}$ value in the films to varying degrees, since they provided the black color to the system typical of carbon, which stayed the same as a function of the CNT concentration, from the preparation of the generating solution of the film until film formation. The aforementioned color is formed by thermal decomposition or incomplete combustion of hydrocarbons [19].

3.2. Moisture and Water Activity $\left(A_{W}\right)$. The moisture content in the films was not significantly different $(P>0.05)$ between samples with varying concentrations of CNTs (Table 1). This is most likely due to the predominant hydrophobic nature and slight hydrophilic nature of the $\mathrm{CNT}$ /gelatin compounds, as a result of the potential interaction of negative sulfonic groups $\left(\mathrm{SO}_{3}{ }^{-}\right)$on the SDS surfactant present in the CNTs, with the positive ammonium group $\left(\mathrm{NH}_{3}{ }^{+}\right)$of the biopolymer and the water molecules. Therefore, there was no observed tendency to hydrate [10]. Moreover, the evaluation of $A_{W}$ in the biodegradable films helps quantitatively predicting its useful life and defines its storage conditions, since the $A_{W}$ represents water available for performing chemical, enzymatic, and microbiological reactions [20]. The integration of the CNT in films showed a slight tendency to increase, even when the 


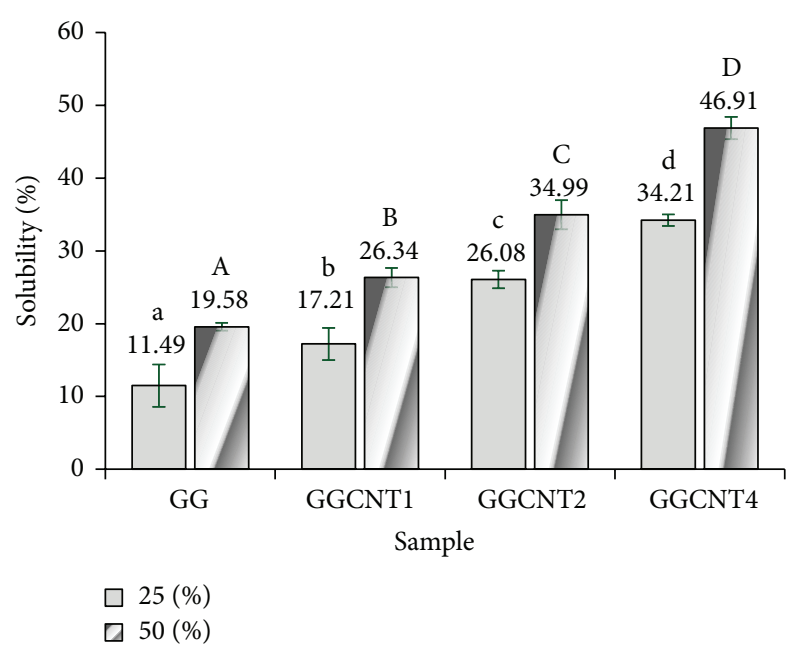

FIGURE 1: Solubility of films from gelatin/glycerol and carbon nanotubes, at $25^{\circ} \mathrm{C}$ and $80^{\circ} \mathrm{C}$. Error bars with the same letter are not significantly different $(P>0.05)$.

$A_{W}$ values were not significant with respect to the control film (Table 1).

3.3. Solubility. Evaluation of the water solubility of the films is an important factor in determining biodegradability potential, since polymers are more susceptible to degradation at higher solubility, because components are easier to hydrolyze and consequently become smaller molecules. The solubility percentage of the film with the highest content of CNTs with respect to the GG film was increased up to 22.72 and $27.33 \%$ in the given solubility at $25.0^{\circ}$ and $80.0^{\circ} \mathrm{C}$, respectively (Figure 1). An increase in temperature provided further hydrolysis of the polymer chains, by increasing the kinetic energy of the components of the films, which resulted in an increase in the percentage of the solubility determined at $80.0^{\circ} \mathrm{C}$. These results suggest that the increase in solubility of the films with CNT is partly due to a certain hydrophilic tendency conferred by the surfactant (SDS), upon directly contacting water at this temperature.

Mahmoud and Savello [21] also observed the increased solubility of protein based films, and this may be explained by the decrease in protein interactions with the other constituents in the polymer matrix. This suggests that the increased concentration of CNT and SDS leads to increases in the interaction with the multicomponent gelatin/glycerol and water system and to better CNT dispersion.

3.4. X-Ray Diffraction. The type B gelatin used for making films in this study showed an X-ray diffraction pattern, characteristic of a partially crystalline material, with a wide maximum (peak) of absorption located at 2-theta: $20^{\circ}$ (Figure 2). According to Tanioka et al. [22], this peak indicates the reconstitution of the collagen triple helix structure. The GG or gelatin/glycerol/SDS control film showed a peak at 2-theta: $8^{\circ}$. This maximum is related to the diameter of the triple helix and its intensity and also could be associated with the triple helix content in the films [23]. The pattern of crystallinity

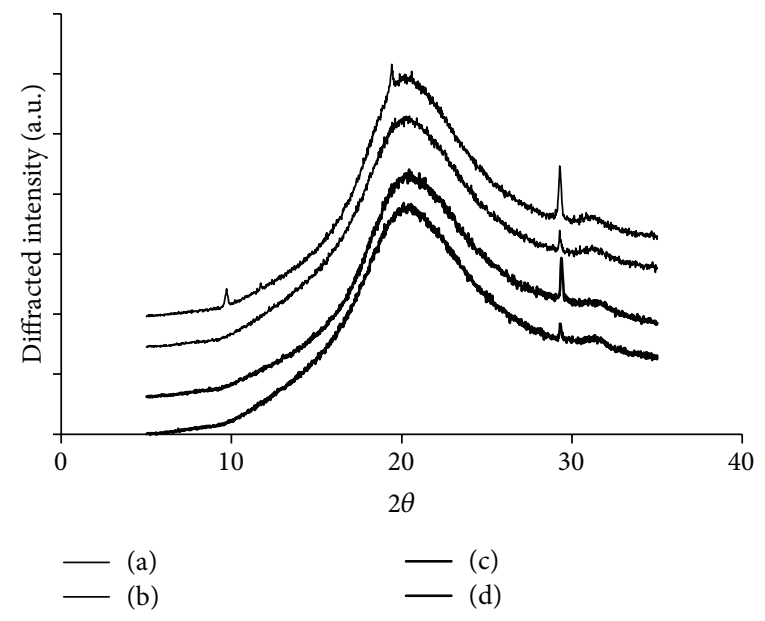

Figure 2: Diffractograms of films from gelatin/glycerol and carbon nanotubes. (a) GG and (b) GGNTC1; (c) GGNTC2 and (d) GGNTC4.

was not observed in the films containing CNTs/SDS/glycerol films, because they did not show any peak at the same intensity $\left(8^{\circ}\right)$, which caused diffractograms with absorption maximums in a larger number of amorphous zones. These findings indicate that the CNTs interfered with the crystalline arrangement of the gelatin triple helix structure, thus causing a depression in the peaks of crystallinity. A slight decrease was observed in the percentage of crystallinity in the films with CNTs as follows: 83.44\% (GGCNT4), 83.53\% (GGCNT2), and $83.83 \%$ (GGCNT1), with respect to the control GG film $(83.84 \%)$. Except for the 1st peak presented by the control sample, no difference was seen in the common maximum intensity presented by the films (2-theta: $20^{\circ}$ ). The signal observed at approximately 2 -theta, $30^{\circ}$, is considered to be noise that is occasionally seen on diffractograms and emitted by the aluminum sample holder.

3.5. Thermal Analysis. The effects of CNTs on the thermal properties of the films are shown in Figure 3. The thermograms obtained showed an endothermic transition characteristic of first order transitions associated with phase changes $\left(T_{f}\right)$. The value of $T_{f}$ for the control film was $156.0^{\circ} \mathrm{C}$; however, this transition temperature decreased as the concentration of CNTs increased. Consequently, the lowest value of $T_{f}$ was from GGCNT4, which was significantly different from the rest of the other samples $(P<0.05)$ (Table 2$)$. This can be attributed to a decrease in crystallinity by the addition of CNTs and is also associated with the lower amount of energy required to break the crystal chain of the material, which resulted in lower values obtained from enthalpy of fusion $(\Delta H, 28.25 \mathrm{~J} / \mathrm{g})[24,25]$.

The results found in this study were similar to those reported by Arvanitoyannis et al. [26] for films prepared from gelatin/starch/glycerol (115.6 to $\left.144.2^{\circ} \mathrm{C}\right)$ and Rivero et al. [27] in gelatin films at different concentrations of glycerol (87.9 to $132.4^{\circ} \mathrm{C}$ ). Arvanitoyannis et al. [26] noted that it is difficult to detect the glass transition in very complex systems, such as 


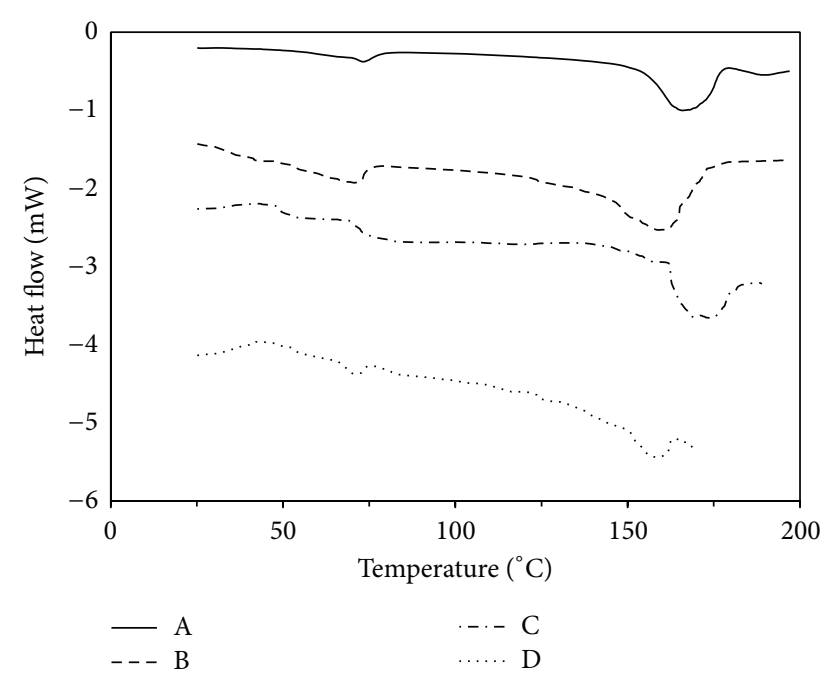

FIGURE 3: Thermal transitions of films from gelatin/glycerol and carbon nanotubes. (A) GG and (B) GGNTC1; (C) GGNTC2 and (D) GGNTC4.

TABLE 2: Thermal parameters of films from gelatin/glycerol and carbon nanotubes* .

\begin{tabular}{lccc}
\hline Sample & $T_{g}\left({ }^{\circ} \mathrm{C}\right)$ & $T_{f}\left({ }^{\circ} \mathrm{C}\right)$ & $\Delta H(\mathrm{~J} / \mathrm{g})$ \\
\hline GG & $72.98 \pm 0.80^{\mathrm{a}}$ & $156.29 \pm 0.28^{\mathrm{a}}$ & $40.40 \pm 0.63^{\mathrm{a}}$ \\
GGNTC1 & $57.26 \pm 2.89^{\mathrm{b}}$ & $153.04 \pm 1.95^{\mathrm{a}}$ & $37.46 \pm 0.88^{\mathrm{a}}$ \\
GGNTC2 & $56.32 \pm 3.20^{\mathrm{b}}$ & $142.95 \pm 1.21^{\mathrm{b}}$ & $34.82 \pm 2.68^{\mathrm{ab}}$ \\
GGNTC4 & $54.62 \pm 0.84^{\mathrm{b}}$ & $132.02 \pm 3.32^{\mathrm{c}}$ & $28.25 \pm 0.99^{\mathrm{b}}$ \\
\hline
\end{tabular}

${ }^{*}$ Mean values of three measurements \pm standard error.

Same letters after entries within each column indicate that there are no significant differences $(P>0.05)$.

gelatin/starch/polyol/water, particularly at low moisture content $(<7.0 \%)$. However, in this study, second order transitions associated with the glass transition temperature $\left(T_{g}\right)$ of the films could be detected. The GG film showed a higher value of $T_{g}\left(72.98^{\circ} \mathrm{C}\right)$ and was significantly different $(P<0.05)$ from samples with CNTs, which showed a $T_{g}$ in the range of 57.26 to $54.64^{\circ} \mathrm{C}$ (Table 2).

Therefore, it can be stated that, once stabilized, the CNTs caused a decrease in molecular mobility and the free volume in the system with respect to the original state, which caused a depression in the $T_{g}$ and had a direct impact on the thermal properties of films.

3.6. Mechanical Properties. As expected, the addition of CNTs modified the mechanical properties of the films with respect to the GG film. A progressive decrease of the fracture tension (FT) was observed as the concentration of CNTs increased (Figure 4(a)). This indicates that, in the gelatin/glycerol system, CNTs were distributed in a partially homogeneous form in certain regions of the polymer matrix, which resulted in a decrease in the resistance or strength required to fracture the film. Moreover, the decrease was generally proportional to the concentration of the CNTs. Similar values of fracture tension were reported for films containing $0.001 \%(\mathrm{w} / \mathrm{w})$ of CNT, at $58 \%$ relative humidity, by Ortiz-Zarama et al. [28].

Elongation at break showed the opposite tendency. It was found that the percent elongation $(\% E)$ was directly proportional to the concentration of the added CNTs and increased up to $26.0 \%$ in the film with a CNT concentration of $0.004 \%$ (Figure $4(\mathrm{~b})$ ). Importantly, this result was significantly different from the other samples $(P<0.05)$. This increase in the extensibility of the films could be because once the CNTs were inserted in the polymer chains of the gelatin, they caused some increase in the state of order, which then caused the aforementioned reduction of $T_{g}$, thus enabling a more ductile and flexible material to be obtained. Rivero et al. [27] reported an increase in values of $\% E$ of $180.0 \%$ in films with a high content of glycerol $(100.0 \% \mathrm{w} / \mathrm{w})$. OrtizZarama et al. [28] found up to $168 \% E$ in films with CNT addition, under controlled relative humidity. These values are similar to the increases found in this study $(166.0 \%)$; however, it is noteworthy that the effect on the $\% E$ in this study is attributed mainly to the CNTs and not to glycerol, since a constant amount $(1.0 \% \mathrm{w} / \mathrm{w})$ of the plasticizer was used in all cases. Moreover, the behavior of the results of the elastic modulus (EM) in the films with CNTs is consistent with that of a softer plastic [29], since EM values decreased as the concentration of CNTs increased (Figure 4(c)). The GG or gelatin/glycerol/SDS control film provided a high EM and therefore would be better classified as hard or compact plastic.

This contention correlates well with the trend observed in the fracture tension, with predominance of the control film, just like high values of $\% E$ found in films of gelatin with CNT/SDS/glycerol. In this regard, it was observed that the GG film showed the typical characteristics of the fragile and rigid materials, since it exhibited high values of TF and EM and low values of $\% E$. Similar trends have been found in gelatin films lacking or with low concentrations of plasticizer [27], as well as in gelatin films with hydroxypropyl starch [4].

3.7. Water Vapor Permeability (WVP). The results of the WVP tests of the films are shown in Figure 5. The GG film showed the lowest value of WVP. This can be attributed to the fact that this control film has a more closed or compact structure compared to the treated samples due to an increased amount of crystalline regions in its structure, which thus restricts the passage of water through it. A decrease of the crystallinity in the GGCNT2 and GGCNT4 films because of the addition of the CNTs caused a significant increase $(P<0.05)$ in the WVP in the films assessed. These results indicate that, at a concentration greater than $0.001 \% \mathrm{w} / \mathrm{w}$, the CNTs conferred a greater hydrophilic degree to the films, thus facilitating the passage of water vapor through its structure, being consistent with the results reported by Ortiz-Zarama et al. [28], who did not find significant differences in films with $0.001 \%$ CNT addition.

Moreover, it is well known that the amorphous areas of the material are subject to water absorption, meaning that the increase in the aforementioned areas in films with CNT/SDS/glycerol likely contributed to the increase in WVP. The values reported here are similar to those obtained by Gennadios et al. [30], based on films made from 


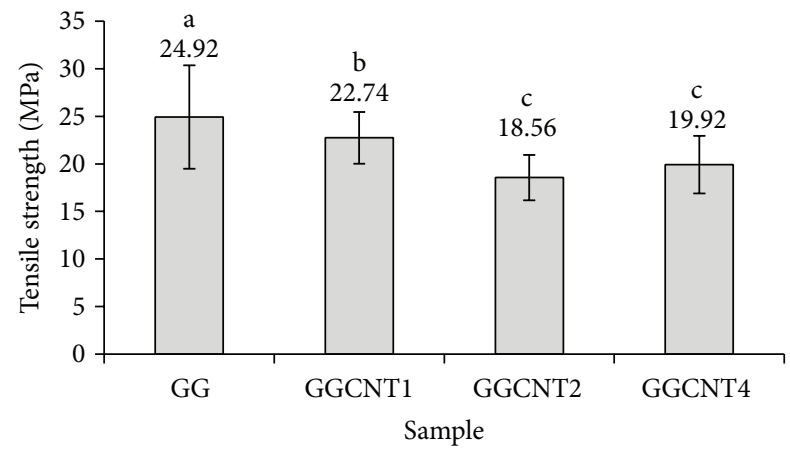

(a)

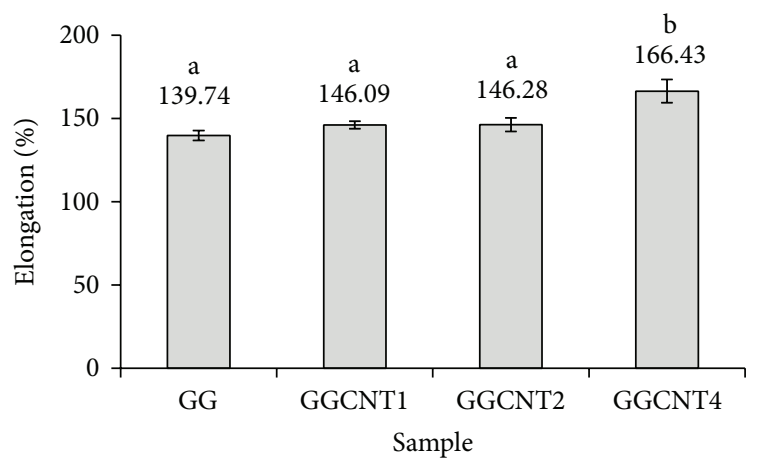

(b)

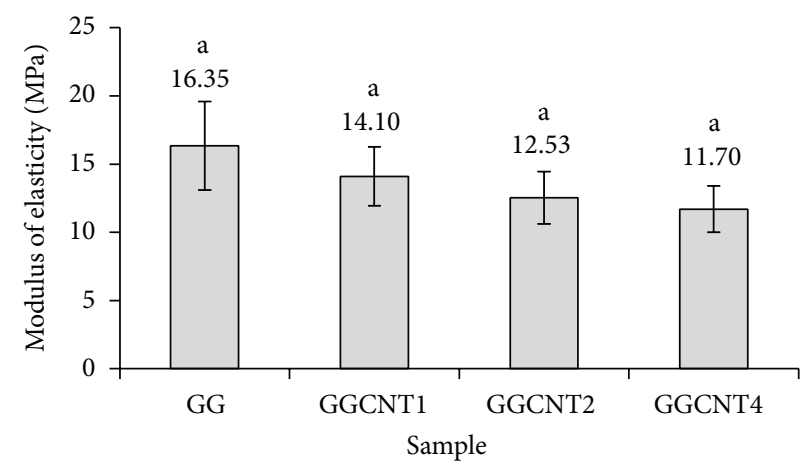

(c)

FIGURE 4: Mechanical properties of films from gelatin/glycerol and carbon nanotubes. Error bars with the same letter are not significantly different $(P>0.05)$.

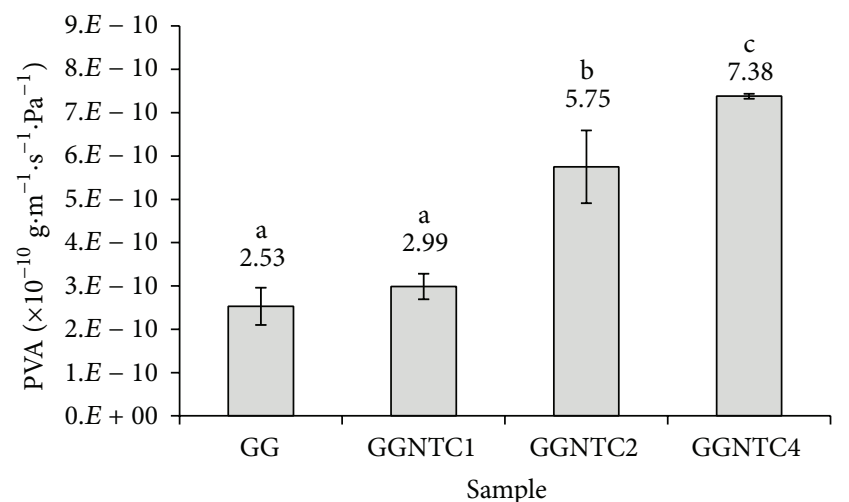

FIGURE 5: Water vapor permeability (WVP) of films from gelatin/glycerol and carbon nanotubes. Error bars with the same letter are not significantly different $(P>0.05)$.

wheat gluten (protein) using glycerol as a plasticizer $(7.0 \times$ $\left.10^{-10} \mathrm{~g} \mathrm{~m}^{-1} \mathrm{~s}^{-1} \cdot \mathrm{Pa}^{-1}\right)$ and those found by Rivero et al. [27] in gelatin films at different concentrations of glycerol $(3.87 \times$ $\left.10^{-10} \mathrm{~g} \mathrm{~m}^{-1} \cdot \mathrm{s}^{-1} \mathrm{~Pa}^{-1}\right)$.

3.8. Image Analysis. Results from image analyses are shown in Figure 6. The surface fractal dimension (SFD), which at values of SFD $=1$ and $\mathrm{SFD}=2$ indicates a regular surface, was also measured. It was found that the gelatin/glycerol/SDS control film showed values close to one (for lack of pixels
TABLE 3: Texture parameters obtained by image analysis of films from gelatin/glycerol and carbon nanotubes*.

\begin{tabular}{lccc}
\hline Sample & DFS & Entropy & $\%$ area \\
\hline GG & $0.983 \pm 0.01^{\mathrm{a}}$ & $7.120 \pm 0.02^{\mathrm{a}}$ & $0.026 \pm 0.01^{\mathrm{a}}$ \\
GGNTC1 & $1.560 \pm 0.02^{\mathrm{b}}$ & $9.212 \pm 0.05^{\mathrm{b}}$ & $18.715 \pm 1.04^{\mathrm{b}}$ \\
GGNTC2 & $1.791 \pm 0.01^{\mathrm{c}}$ & $10.189 \pm 0.01^{\mathrm{c}}$ & $34.168 \pm 0.43^{\mathrm{c}}$ \\
GGNTC4 & $1.886 \pm 0.01^{\mathrm{d}}$ & $10.414 \pm 0.02^{\mathrm{d}}$ & $44.795 \pm 0.43^{\mathrm{d}}$ \\
\hline
\end{tabular}

${ }^{*}$ Mean values of three measurements \pm standard error.

Same letters after entries within each column indicate that there are no significant differences $(P>0.05)$.

in the image). However, the GGCNT or CNT/SDS/glycerol film showed values of $S F D=1.56$, which suggested an uneven surface. This was not the case for films with higher concentrations of nanotubes; GGCNT2 and GGCNT4 showed SFD values of 1.79 and 1.88 , respectively, showing evidence of a smoother surface or with a higher order $(\mathrm{SFD}=2)$. The SFD showed high correlation coefficients with respect to the mechanical parameters of the FT voltage $(r=0.91)$ and elastic modulus (EM; $r=0.98$ ), suggesting that the increase in the CNT concentration made the gelatin polymer matrix have a more regular surface and, consequently, allowed for more elastic films (higher elongation). These results are consistent with the results of the mechanical tests.

Moreover, the disorder (entropy) in the images obtained in the films (Table 3) was quantified in pixels. All samples 


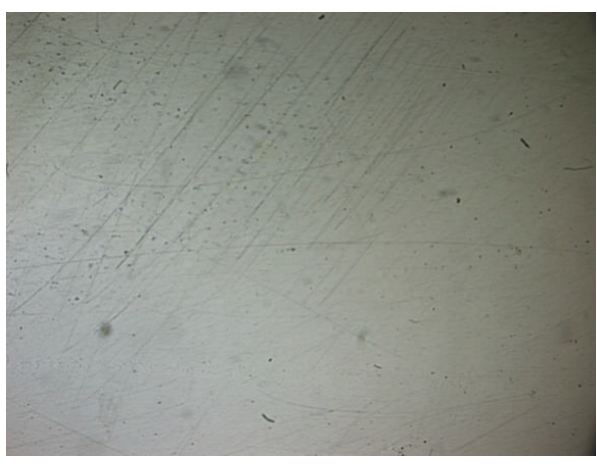

(a)

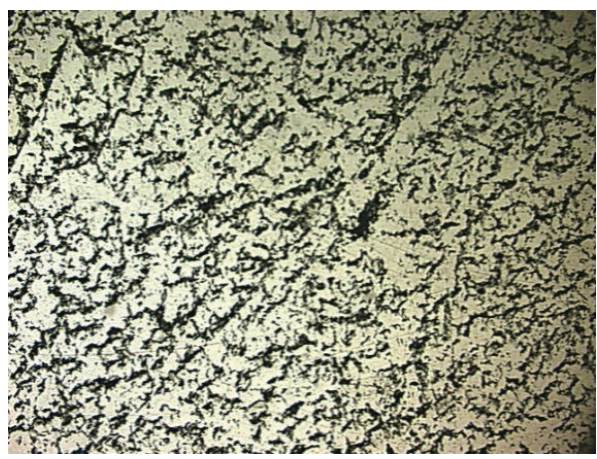

(c)

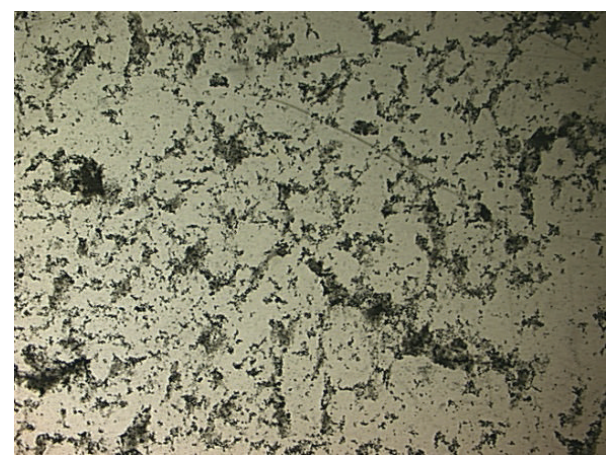

(b)

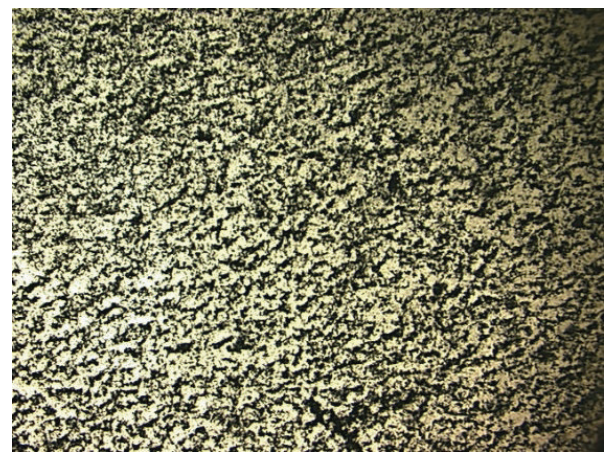

(d)

FIGURE 6: Images of films from gelatin/glycerol and carbon nanotubes. (a) GG and (b) GGNTC1; (c) GGNTC2 and (d) GGNTC4.

had statistically significant $(P<0.05)$ differences between each other, and the entropy value increased as the CNT concentration increased. The results also correlated with the percentage of crystallinity and the fusion temperature of the films, which were $r=0.93$ and $r=0.99$, respectively. Based on the image analyses and the structural arrangement of the films, it can be inferred that a higher concentration of CNTs produced a structure with a greater number of amorphous regions, which consequently led to a decrease in the percentage of crystallinity and the fusion temperature of the films. Finally, the percentage of area $(\% A)$ covered by CNTs in the gelatin polymer matrix was calculated, and we found that GGCNT2 and GGCNT1 had a lower $\% A$ compared to the GGCNT4 film.

Thus, the highest concentration of CNTs $(0.004 \% \mathrm{w} / \mathrm{w})$ most likely had the greatest area of contact with the polymer matrix, which resulted in the most significant changes to the physical, thermal, mechanical, and barrier properties of the films, suggesting a nanotube network formation in the protein matrix.

\section{Conclusions}

A method readily available, to prepare glycerol/gelatin based films, by doping the film with a low concentration of carbon nanotubes and sodium dodecyl sulfate, was essayed. CNT/SDS/glycerol films of gelatin presented darker color, indicating that they could be applied to sensitive products that need to be protected from physical factors, such as light. Those films with CNTs inclusion also showed greater values of solubility and water activity, with $30 \%$ increase in elongation at break in gelatin/glycerol/SDS films with $0.004 \%$ CNT, with respect to gelatin/glycerol/SDS control films. Moreover, the decrease of crystallinity in CNT/SDS/glycerol films caused a decrease in $T_{g}$, which was associated with a change in the molecular mobility in the system. The addition of more than $0.001 \%$ of CNTs decreased the FT of gelatin films, with increase in extensibility. However, the EM of gelatin/CNT/SDS/glycerol films was not significantly affected because of the CNTs addition, resulting in films classified as soft. Nonetheless, the $T_{f}$ and $\Delta H$ of such films decreased, causing an increase in the WVP due to an increase in the amorphous regions. The films fractal dimension indicated that the inclusion of the highest concentration of CNT gave place to films with a homogeneous surface, suggesting a nanotube network formation in the protein matrix. Further research is needed to widen the knowledge concerning the interactions of gelatin matrix CNTs, but, according to the results, this work has shown that the easy method used to prepare CNTs/gelatin/glycerol/SDS composite materials produces films with overall better physical, mechanical, and thermal properties.

\section{Conflict of Interests}

The authors declare that there is no conflict of interests regarding the publication of this paper. 


\section{Acknowledgments}

The authors are grateful to Instituto Politécnico Nacional (Projects 20141318 and 20150956), CONACyT, SNI, COFAA, and EDI in Mexico.

\section{References}

[1] G. Espín, Plásticos y Contaminación Ambiental, Universidad Nacional Autónoma de México, Mexico City, México, 2007.

[2] K. Y. Lee, J. Shim, and H. G. Lee, "Mechanical properties of gellan and gelatin composite films," Carbohydrate Polymers, vol. 56, no. 2, pp. 251-254, 2004.

[3] V. Pandey, N. Mehta, S. K. Tripathi, and A. Kumar, "Optical band gap and optical constants in $\mathrm{Se}_{85} \mathrm{Te}_{15-x} \mathrm{~Pb}_{x}$ thin films," Chalcogenide Letters, vol. 2, no. 5, pp. 39-44, 2005.

[4] I. Arvanitoyannis, A. Nakayama, and S.-I. Aiba, "Edible films made from hydroxypropyl starch and gelatin and plasticized by polyols and water," Carbohydrate Polymers, vol. 36, no. 2-3, pp. 105-119, 1998.

[5] N. Cao, X. Yang, and Y. X. Fu, "Effects of various plasticizers on mechanical and water vapor barrier properties of gelatin films," Food Hydrocolloids, vol. 23, no. 3, pp. 729-735, 2009.

[6] R. N. Tharanathan, "Biodegradable films and composite coatings: past, present and future," Trends in Food Science and Technology, vol. 14, no. 3, pp. 71-78, 2003.

[7] S. E. Sánchez, Research on preparation of carbon nanotube dispersions in aqueous media [BSc Thesis], Graduate School of Biochemical Engineering, Technological Institute of Durango, 2007.

[8] S. Iijima, "Helical microtubules of graphitic carbon," Nature, vol. 354, no. 6348, pp. 56-58, 1991.

[9] D. Qian, E. C. Dickey, R. Andrews, and T. Rantell, "Load transfer and deformation mechanisms in carbon nanotube-polystyrene composites," Applied Physics Letters, vol. 76, no. 20, pp. 28682870, 2000.

[10] S. Haider, S.-Y. Park, K. Saeed, and B. L. Farmer, "Swelling and electroresponsive characteristics of gelatin immobilized onto multi-walled carbon nanotubes," Sensors and Actuators B: Chemical, vol. 124, no. 2, pp. 517-528, 2007.

[11] M. A. García, A. Pinotti, M. N. Martino, and N. E. Zaritzky, "Characterization of composite hydrocolloid films," Carbohydrate Polymers, vol. 56, no. 3, pp. 339-345, 2004.

[12] AOAC, Official Methods of Analysis, Association of Official Analytical Chemists, Washington, DC, USA, 10th edition, 1980.

[13] K. Hayakawa, K. Tanaka, T. Nakamura, S. Endo, and T. Hoshino, "Quality characteristics of waxy hexaploid wheat (Triticum aestivum L.): properties of starch gelatinization and Retrogradation," Cereal Chemistry, vol. 74, no. 5, pp. 576-580, 1997.

[14] J. T. Manful, C. C. Grimm, J. Gayin, and R. D. Coker, "Effect of variable parboiling on crystallinity of rice samples," Cereal Chemistry, vol. 85, no. 1, pp. 92-95, 2008.

[15] ASTM 882-95a, Standard Test Methods for Tensile Properties of Thin Plastic Sheeting, American Society for Testing and Materials, West Conshohocken, Pa, USA, 1995.

[16] ASTM E96-80, Standard Test Methods for Water Vapour Transmission of Materials in Sheet Form, American Society for Testing and Materials, West Conshohocken, Pa, USA, 1980.

[17] D. C. Montgomery, Design and Analysis of Experiments, John Wiley \& Sons, New York, NY, USA, 2006.
[18] F. Vazquez, Study of physical properties of films prepared from fish gelatin [BSc thesis], Graduate School, University of La Frontera, 2005.

[19] M. Doerner, The Materials of the Artist and Their Use in Painting: With Notes on the Techniques of the Old Masters, Harcourt, London, UK, 1984.

[20] M. S. Rahman and S. S. Sablani, Water Activity Measurement Methods of Foods, CRC Press, London, UK, 2009.

[21] R. Mahmoud and P. A. Savello, "Solubility and hydrolyzability of films produced by transglutaminase catalytic crosslinking of whey protein," Journal of Dairy Science, vol. 76, no. 1, pp. 29-35, 1993.

[22] A. Tanioka, K. Miyasaka, and K. Ishikawa, "Reconstitution of collagen fold structure with stretching of gelatin film," Biopolymers, vol. 15, no. 8, pp. 1505-1511, 1976.

[23] A. Bigi, S. Panzavolta, and K. Rubini, "Relationship between triple-helix content and mechanical properties of gelatin films," Biomaterials, vol. 25, no. 25, pp. 5675-5680, 2004.

[24] J. Jane, Y. Y. Chen, L. F. Lee et al., "Effects of amylopectin branch chain length and amylose content on the gelatinization and pasting properties of starch," Cereal Chemistry, vol. 76, no. 5, pp. 629-637, 1999.

[25] J. Patindol and Y.-J. Wang, "Fine structures of starches from long-grain rice cultivars with different functionality," Cereal Chemistry, vol. 79, no. 3, pp. 465-469, 2002.

[26] I. Arvanitoyannis, E. Psomiadou, A. Nakayama, S. Aiba, and N. Yamamoto, "Edible films made from gelatin, soluble starch and polyols. Part 3," Food Chemistry, vol. 60, no. 4, pp. 593-604, 1997.

[27] S. Rivero, M. A. García, and A. Pinotti, "Correlations between structural, barrier, thermal and mechanical properties of plasticized gelatin films," Innovative Food Science \& Emerging Technologies, vol. 11, no. 2, pp. 369-375, 2010.

[28] M. A. Ortiz-Zarama, A. Jiménez-Aparicio, M. J. Perea-Flores, and J. Solorza-Feria, "Barrier, mechanical and morpho-structural properties of gelatin films with carbon nanotubes addition," Journal of Food Engineering, vol. 120, no. 1, pp. 223-232, 2014.

[29] S. Vishu, Handbook of Plastics Testing Technology, John Wiley \& Sons, New York, NY, USA, 1983.

[30] A. Gennadios, C. L. Weller, and C. H. Gooding, "Measurement errors in water vapor permeability of highly permeable, hydrophilic edible films," Journal of Food Engineering, vol. 21, no. 4, pp. 395-409, 1994. 

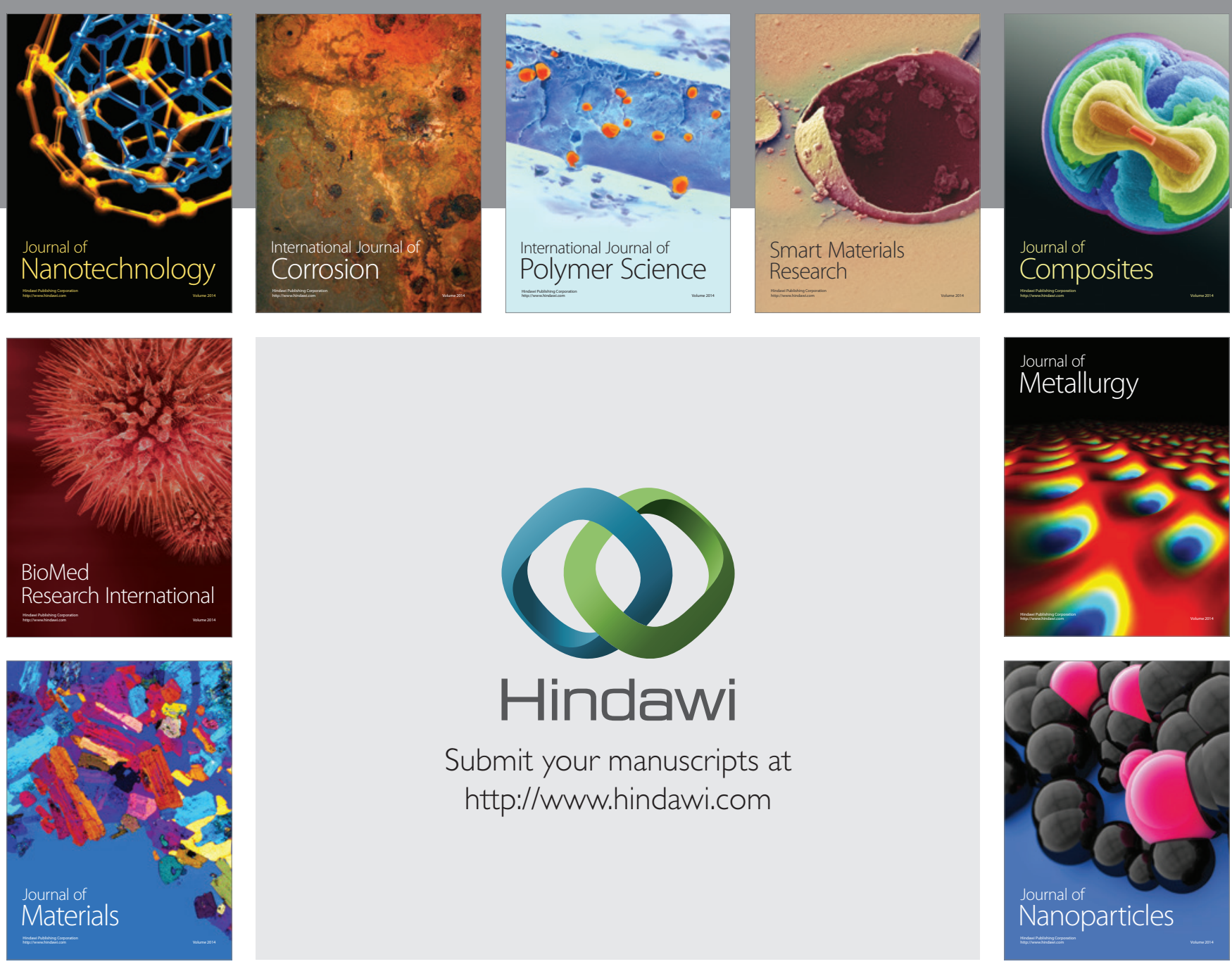

Submit your manuscripts at http://www.hindawi.com
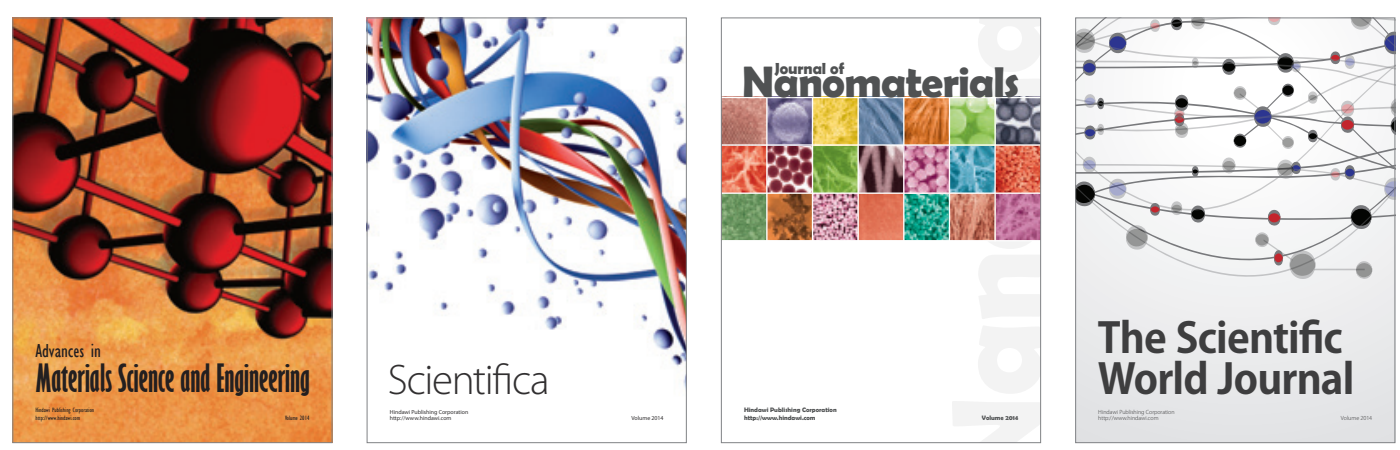

\section{The Scientific World Journal}
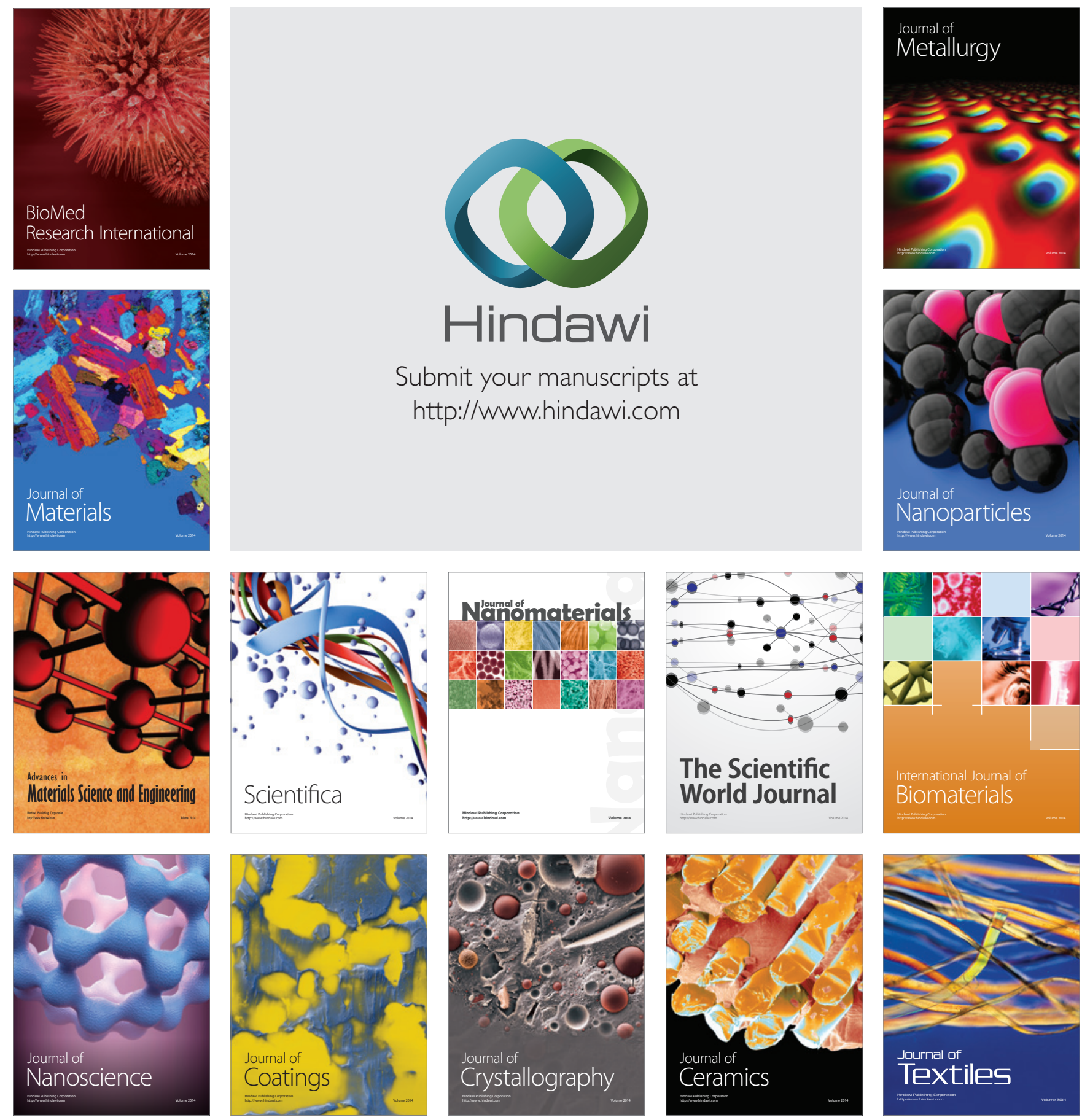